\title{
Competencia comunicativa y nuevas tecnologías en el proceso de localización web: modelo MDPT para la formación de profesionales en localización
}

\author{
Communicative Competence and ICT in the Process of Website \\ Localization: PATT Model for Localization and Translation \\ Training
}

Recibido: 06/04/2018

Juncal Gutiérrez-Artacho

María-Dolores Olvera-Lobo

Irene Rivera-Trigueros

Universidad de Granada

Aceptado: 17/12/2018

\begin{abstract}
Accessing external markets has become a priority for companies given the constant changes in the economic environment boosted by globalization. Website localization constitutes a new field of study and professional intervention within the translation field. Localization is the process of adapting a website to the typological, discursive and genre conventions of the target language adapting that website to a different language and culture. Undoubtedly, excellent language skills are essential for the translation process. However, website localization entails much more than simply translating the content of the pages. In the localization process the target culture must be taken into account; therefore, a full communicative competence -covering linguistic, sociolinguistic and pragmatic subcompetences-is essential. In addition, the content of a website is made up of text, images and other multimedia elements, all of which have to be translated and subjected to cultural adaptation thanks to technology tools. As a result, having a mastery of ICT is another key element in the localization process. This contribution presents the application of a new teaching methodology to the teaching/learning of localization in the BA in Translation and Interpreting at Universidad de Granada, applying the PATT (Professional Approach to Translation Training) model. The model has been revised and redesigned to integrate the communicative competence as well as the technological features required by this emerging professional profile.
\end{abstract}

KEY WORDS: Website Localization, Translation Training, Communicative Competence, ICT

\section{RESUMEN}

El acceso a mercados exteriores se ha convertido en una prioridad para las empresas dados los continuos cambios en el entorno económico impulsados por la globalización. La localización web se ha perfilado en la actualidad como un nuevo campo de estudio y de intervención profesional dentro del ámbito de la traducción. La localización se encarga de adaptar un sitio web a las convenciones de género, tipológicas y discursivas de la lengua meta, es decir, se trata de adaptar un sitio web a una lengua y cultura diferente. Sin duda, un excelente componente lingüístico es esencial para poder llevar a cabo el proceso traslativo. Sin embargo, la localización va más allá de la traducción. En el proceso de localización entra en juego la cultura meta, por tanto, una competencia comunicativa completa - que abarque los componentes lingüístico, sociolingüístico y pragmático- es imprescindible. Además, el contenido de un sitio web está formado por texto, imágenes y otros elementos multimedia que deben ser traducidos y adaptados culturalmente haciendo uso de diferentes recursos tecnológicos, por lo que el dominio de las nuevas tecnologías es otra pieza clave del proceso de localización. Este trabajo presenta la aplicación de una nueva metodología para la enseñanza/aprendizaje de la localización por parte de estudiantes del Grado en Traducción e Interpretación en la Universidad de Granada, basada en la aplicación del modelo didáctico MPDT (Modelo Profesional para la Didáctica de la Traducción). Se ha revisado y rediseñado este modelo para integrar tanto la competencia comunicativa completa como las nuevas habilidades tecnológicas que requiere este nuevo perfil profesional.

PALABRAS CLAVE: localización, localización web, enseñanza de la traducción, innovación docente

Authors' correspondence address:

Juncal Gutiérrez-Artacho, Departamento de Traducción e Intrepretación. Universidad de Granada. Granada, España. ORCID 0000-0002-0275-600X. E-mail: juncalgutierrez@ugr.es.

María-Dolores Olvera-Lobo, Departamento de Información y Comunicación. Universidad de Granada. Granada, España. ORCID 0000-0002-0489-7674

Irene Rivera-Trigueros, Departamento Traducción e Interpretación. Universidad de Granada, España. ORCID 0000-0003-4877-4083 


\section{Introducción}

La localización forma parte de un proceso global, denominado GILT, que incluye la globalización, la internacionalización, la localización y la traducción (Arevalillo-Doval, 2000; LISA, 2003). La globalización combina la internalización y la localización para diseñar sitios web que se puedan adaptar a diferentes países realizando los mínimos cambios necesarios; la internacionalización se encarga del diseño de un sitio web para que se pueda adaptar a culturas específicas, es decir, se aíslan los elementos propios de un idioma y cultura para facilitar su adaptación; la localización es el proceso de adaptar un sitio web para que parezca propio de un mercado local específico conocido como locale; $\mathrm{y}$, finalmente, la traducción consiste en comprender el significado de un texto en una lengua origen para producir un texto con significado equivalente en la lengua meta o de destino (Cadieux y Esselink, 2004; Corte, 2002).

La implementación de estos procesos, a pesar de ser complejos y costosos, es clave para las empresas a la hora de abrirse a mercados exteriores (Corte, 2002). En consecuencia, la internalización y localización de páginas web se ha convertido en un elemento esencial para las empresas de cara a acceder a estos mercados y aumentar así sus beneficios. Como consecuencia, la localización web se ha perfilado en los últimos años como un nuevo campo de estudio y de intervención profesional dentro del ámbito de la traducción.

El mercado de trabajo al que acceden los egresados y las egresadas del Grado en Traducción e Interpretación supone un desafío constante que demanda continuamente nuevos perfiles profesionales y relaciones interdisciplinares con otras áreas de conocimiento. Se trata, por tanto, de un mercado global, descentralizado, virtual y exigente (Hussein, 2007; Olvera-Lobo et al., 2005). El nuevo perfil del profesional en localización es un claro ejemplo de interdisciplinariedad, ya que precisa de una competencia comunicativa completa junto con destrezas tecnológicas que le permitan dominar herramientas y recursos específicos empleados en la localización. Ante la situación que venimos relatando, las metodologías docentes también deben evolucionar y adaptarse a este nuevo panorama para salvar las distancias existentes en la actualidad entre la práctica profesional y el ámbito académico (Gaspari et al., 2015).

En este trabajo se presenta el Modelo Profesional para la Didáctica de la Traducción (MPDT) (Gutiérrez-Artacho y Olvera-Lobo, 2013, 2016, 2017a, 2017b, 2018; Olvera-Lobo et al., 2005, 2007a, 2007b, 2008, 2009a, 2009b, 2010, 2011, 2013, 2014a, 2014b, 2014c, 2016, 2017a, 2017b; Robinson et al., 2015, 2016a, 2016b) revisado y adaptado a las nuevas necesidades de este perfil profesional de modo que aúne la competencia comunicativa completa y el uso de las TIC ofreciendo un entorno de enseñanza-aprendizaje innovador, en sintonía con la realidad de la generación de nativos y nativas digitales, que contribuya a facilitar la adquisición de competencias por parte de los estudiantes y a incrementar su compromiso respecto a su formación (Olvera-Lobo y Gutiérrez-Artacho, 2017a, 2017b).

\subsection{Diferencia entre la traducción y la localización}

Los inicios de la localización se sitúan a finales de los años 80, cuando comienzan a llevarse a cabo las primeras traducciones de softwwares. Los conocimientos técnicos e informáticos se convirtieron en esenciales para aquellos traductores que querían adentrarse en esta nueva disciplina (GarcíaSaavedra, 2002). Indudablemente, la localización conlleva un proceso de traducción, sin embargo, esta tarea también incluye la adaptación a la cultura meta de elementos tales como audios, vídeos, imágenes, iconos, colores o formatos de fecha, entre otros. Por todo ello, requiere de conocimientos y técnicas que no son puramente lingüísticas y es necesario, además, contar con herramientas informáticas especializadas para poder llevar a cabo el proceso de localización de manera eficiente (Anastasiou, 2010; Arevalillo, 2000; García-Saavedra, 2002).

Por otra parte, el proceso de traducción conlleva que un profesional lingüístico lleve a cabo varios roles y diferentes tareas para completar cada fase del proceso a tiempo y acorde con los parámetros de calidad determinados en el encargo de traducción (Olvera-Lobo et al., 2007a, 2007b, 2008, 2009, 2011, 2014; Robinson et al., 2015, 2016a, 2016b). En contraposición, por lo general, el proceso de localización incluye a diferentes agentes y el propio profesional no permanece aislado, 
forma parte de un equipo en el que se incluyen los directores generales, el/la responsable de localización, desarrolladores, inspectores de calidad, y así como profesionales en ingeniería lingüística, terminología, traducción, entre otros (García-Saavedra, 2002; Gutiérrez-Artacho y Olvera-Lobo, 2016).

\subsection{Formación para profesionales en localización}

La localización se ha perfilado en los últimos años como una nueva dimensión que debe incorporarse a los estudios de traducción dando lugar a una convergencia beneficiosa para ambos ámbitos (Sandrini 2005).

Como consecuencia, la formación reglada sobre la localización ha proliferado en España en los últimos años y en la actualidad se puede encontrar una oferta variada de estudios de posgrado relacionados con este ámbito. Así, ISTRAD (Instituto Superior de Estudios Lingüísticos y Traducción) ofrece dos másteres en los que se pone énfasis en las técnicas y las aplicaciones informáticas empleadas en la localización. Uno de estos programas es el Máster en Traducción Audiovisual: Localización, Subtitulación y Doblaje, ofrecido junto con la Universidad de Cádiz. El otro programa es el máster en Traducción y Nuevas Tecnologías: Traducción de Software y Productos Multimedia y es un título en colaboración con la Universidad Internacional Menéndez Pelayo y Amergin (Instituto Universitario de Estudios Irlandeses de la Universidade A Coruña). Por su parte, en Madrid, la Universidad Autónoma y la Universidad Complutense ofrecen un máster interdisciplinario en Traducción Audiovisual y Localización.

Por otro lado, tanto el máster Tradumática: Tecnologías de la Traducción de la Universidad Autónoma de Barcelona como el máster en Traducción Multimedia de la Universidad de Vigo, el máster en Traducción Profesional de la Universidad de Granada y el máster Doblaje, Traducción y Subtitulación de la Universidad Europea de Madrid cuentan con un módulo compuesto dedicado a la localización.

Para finalizar, la Universidad Alfonso X el Sabio ofrece con un título de Experto en Tradumática, Localización y Traducción Audiovisual e ISTRAD oferta un curso de Experto en Traducción y Localización de Videojuegos.

En cuanto a los aspectos que deberán tenerse en cuenta en la formación para los profesionales de la localización, Drouin (2006) considera que las TIC y los recursos tecnológicos para la localización deben ser una parte clave en el currículum de las enseñanzas de traducción, sin embargo, habrá que tener en cuenta que estas herramientas evolucionan constantemente, por lo que no se trata de enseñar a los y las estudiantes a usarlas sino de tratar de que adquieran consciencia tanto de sus numerosas posibilidades como de sus limitaciones. Además, señala que en su institución, la Universidad de Montreal, hay dos programas que incluyen la localización para estudios de grado, dirigido a estudiantes formados en el ámbito de la informática o en el de la gestión de proyectos en el que se introduce de manera general la localización y se les da a los estudiantes la oportunidad de especializarse a través de cursos relacionados con proyectos de localización; y otro para estudios de posgrado, donde se trata de manera específica la localización y sus herramientas. Además, Drouin (2006) señala la importancia de que la localización se introduzca desde las primeras etapas de formación para que los estudiantes se familiaricen con ella cuanto antes.

Por su parte, O’Hagan (2006), considera es necesario añadir al menos una visión general de la industria de la localización en los currículums de las enseñanzas de traducción. Además de familiarizar a los y las estudiantes con las herramientas tecnológicas empleadas en el ámbito de la traducción los y las estudiantes deben tener un conocimiento básico de informática que incluya la gestión de diferentes tipos de archivos, etiquetas, entre otros. Esta autora también establece que se debe diferenciar la formación de los y las localizadores en función de los diferentes perfiles profesionales (gestor de proyectos, ingenieros de localización, etc.) siempre teniendo en cuenta que la localización surge de aunar la informática con la traducción. En cuanto al tipo de programa puesto en marcha en su institución, Dublin City University, indica que la localización se ofrece como una asignatura opcional en un programa de posgrado sobre traducción, no obstante, precisa que hay una asignatura 
previa sobre tecnologías de traducción que es obligatoria y que permite adquirir una base de conocimientos técnicos además de presentar una visión general de la localización.

Austermühl (2006) propone integrar la localización en la formación de traductores diferenciando tres áreas diferentes: en primer lugar, la traducción para la localización, que se centrará en la localización web y de softwares y que se impartirá en las clases tradicionales de traducción; por otro lado, las herramientas tecnológicas, que se impartirán en clases optativas y comprenden aspectos generales como el uso de los corpus electrónicos, de las memorias de traducción, gestores terminológicos, y aspectos específicos como herramientas empleadas para localización web y de softwares; y, finalmente, la teoría sobre la localización, donde se impartirán las consideraciones teóricas tanto sobre la localización y como sobre los nuevos cambios en las industria de la traducción y los nuevos flujos de trabajo en el ámbito de la localización.

Pym (2013) sugiere que desde las instituciones académicas se debe informar a los estudiantes sobre las nuevos perfiles que surgen en la industria la localización y, si es posible, se les debe formar para que adquieran competencias de gestión que les permitan adaptarse a estos nuevos ámbitos laborales, por tanto, habrá que abrir líneas de comunicación con la industria para que se obtengan beneficios tanto en el ámbito académico como en el profesional. Por otro lado, el trabajo en equipo y la gestión de proyectos tienen que convertirse en partes esenciales de la formación de traductores, además, debería haber transversalidad con otros campos como el del derecho, la informática, etc. Por último, los programas de investigación de las instituciones académicas deberían buscar establecer contratos de colaboración a largo plazo de modo que tanto la industria como los estudiantes y las instituciones académicas puedan obtener un beneficio mutuo.

Por tanto, y en línea con la visión de los autores anteriores, en las enseñanzas de traducción deberán establecerse objetivos curriculares basados en unas competencias cuyo fin sea lograr una transferencia excelente del contenido original al contenido meta teniendo siempre en cuenta que la esencia de la localización reside en aunar los aspectos lingüísticos y culturales -comprendidos en la competencia comunicativa completa- y los aspectos tecnológicos (Dunne, 2006, Jiménez-Crespo y Tercedor, 2012).

\subsection{Competencia comunicativa completa}

A la hora de llevar a cabo el proceso de localización web no basta solo con tener un buen dominio de la lengua meta, al entrar en juego los aspectos culturales es imprescindible que los traductores posean una competencia comunicativa completa - que abarque la competencia lingüística, sociolingüística, discursiva, estratégica y sociocultural-.

El Marco común europeo de referencia para las lenguas (2001) considera que la competencia comunicativa comprende tres componentes que a su vez implican una serie de conocimientos y habilidades. A continuación, se detalla cada uno de ellos a partir de la información proporcionada por dicho estándar europeo.

\subsubsection{Componente lingüistico}

El componente lingüístico o competencia lingüística está relacionado tanto con el alcance y calidad de los conocimientos como la con la organización cognitiva de los mismos y su accesibilidad. Este componente está ligado a las siguientes competencias:

- Competencia léxica: conocimiento sobre el vocabulario de una lengua así como la capacidad de utilizarlo. Está compuesta de los elementos léxicos, comprendidos por las expresiones hechas y la polisemia, y los gramaticales, que pertenecen a clases cerradas de palabras.

- Competencia gramatical: abarca el conocimiento de los recursos gramaticales de una lengua así como la capacidad de usarlos. Es decir, se trata de la capacidad de comprender y expresar significados a través de frases y oraciones bien formuladas y de acuerdo con los principios que rigen la gramática de la lengua implicada. 
- Competencia semántica: abarca la conciencia y el control de la organización del significado. Se divide en la semántica léxica, relacionada con el significado de las palabras; la semántica gramatical, que trata del significado de los elementos, las categorías, las estructuras y los procesos gramaticales; y la semántica pragmática, que se ocupa de las relaciones lógicas.

- Competencia fonológica: conocimientos y destrezas relacionados con la percepción y producción de unidades de sonido, rasgos fonéticos, composición fonética de las palabras y oraciones y la reducción fonética.

- Competencia ortográfica: conocimientos y destrezas en la percepción y producción de los símbolos que componen los textos escritos. Incluyen aspectos como el uso de los signos de puntuación, las convenciones tipográficas, el uso de mayúsculas y minúsculas, entre otros.

- Competencia ortoépica: conocimientos y destrezas relacionados con la articulación de una pronunciación correcta a partir de la forma escrita.

\subsubsection{Componente sociolingüistico}

El componente sociolingüístico está estrechamente ligado al conocimiento sociocultural, es decir, al conocimiento de la sociedad y de la cultura de una determinada comunidad de habla.

Por tanto, este componente hace referencia a la capacidad de utilizar la lengua siguiendo los marcos de conocimientos propios de una comunidad de habla. Al abarcar la dimensión social del uso de una lengua incluye aspectos relacionados con los marcadores lingüísticos de relaciones sociales como la elección del saludo y la forma de tratamiento, las convenciones para los turnos de palabra y las interjecciones; las normas de cortesía; las expresiones de la sabiduría popular, en las que se incluyen los refranes, los modismos y las comillas coloquiales; las diferencias de registro, que podrá ser solemne, formal, neutral, informal, familiar o íntimo; y el dialecto y el acento, que ofrecen importantes claves sobre las características de los interlocutores como su clase social, su procedencia regional o su grupo profesional, entre otros.

\subsubsection{Componente pragmático}

El componente pragmático engloba tres competencias:

- Competencia discursiva: capacidad de ordenar, secuenciar y organizar los mensajes para producir fragmentos coherentes. Esta competencia tendrá en cuenta factores como la temática del texto, la secuenciación temporal, las relaciones de causa-efecto y la organización textual

- Competencia funcional: uso del discurso hablado y de los textos escritos con fines funcionales concretos. Esta competencia incluye las microfunciones, que incluyen categorías de uso funcional de enunciados aislados como persuasión u ofrecer y buscar información factual, entre otros; las macrofunciones, que incluyen categorías de uso funcional de una secuencia de oraciones como la descripción, la narración, la exposición, la argumentación, etc.

- Competencia organizativa: secuenciación de los mensajes en función de los modelos de interacción social de la comunicación.

\subsection{Aspectos técnicos de la localización}

En la actualidad, el dominio de las nuevas tecnologías -y en particular de aquellas específicas para la traducción y la localización- es clave a la hora de formar a los y las estudiantes para su salida al mercado profesional (Gaspari et al., 2015). La formación de los y las localizadores web no solo abarca aquellas tecnologías relacionadas con el ámbito de la traducción, como el dominio de memorias de traducción, bases de datos terminológicas, softwares de traducción asistida o automática, entre otros, sino que además requiere una serie de destrezas específicas. 
En primer lugar, los y las profesionales de la localización se deberán familiarizar con lenguajes de marcado como HTML o XML puesto que la mayoría de páginas web se programan usando este tipo de lenguajes y, aunque un profesional no modificará el código de programación sí que, en ocasiones, deberá modificar el código de etiquetado, por lo que un conocimiento básico de este tipo de lenguajes resultará esencial. Además, en este punto entra en juego la internacionalización y la colaboración interdisciplinar. Si una web ha sido desarrollada aislando los elementos que se deben traducir y localizar del resto del código fuente será mucho más sencillo llevar a cabo el proceso de localización (Pérez-Cabrera, 2012).

Por otra parte, en la actualidad existen herramientas y softwares específicos que permiten separar aquellos contenidos de la web que se deben traducir y localizar, y aislar aquellas cadenas de código que no deben modificarse. La mayoría de estas herramientas emplean el formato XLIFF (Localization Interchange File Format), empleado para estandarizar la localización, por lo que también será necesario que los y las localizadores estén familiarizados con el mismo.

El estándar XLIFF se crea tras el consenso de empresas dedicadas a la localización y la globalización como Novell, Oracle, Sun y Berlitz, entre otras, que se integraron dentro del consorcio OASIS (Organization for the Advancement of Structured Information Standards) (XLIFF OASIS, 2003). El formato XLIFF define a través de vocabularios XML una especificación extensible para el intercambio de información de localización. Este formato permite marcar y localizar aquellos datos que se tienen que localizar y separarlos de las cadenas de código que forman parte de la estructura o skeleton del sitio web. Así, un documento con formato XLIFF consiste, por un lado, en una colección de unidades de traducción, formadas por oraciones o párrafos, del documento origen junto con sus equivalentes en la lengua meta, que serán añadidos por los traductores. Por otro lado, encontramos los elementos que forman parte de la estructura y que no hay que traducir. Una vez realizada la traducción, se unen ambos contenidos formando un documento final.

Este formato permite interoperar con diferentes procesos o fases sin perder información y permite la cooperación entre todos los miembros del equipo desde los y las desarrolladores hasta los traductores y traductoras y los y las localizadores. Además, se pueden reutilizar traducciones de proyectos anteriores, ya que se puede integrar con memorias de traducción. Así, dado que un mismo formato es suficiente para traducir y localizar diferentes tipos de documentos y se puede usar en numerosos softwares específicos de localización, los y las localizadores y traductores pueden elegir la herramienta que consideren más adecuada (Raya, 2004a; Raya, 2004b).

\section{Modelo MPDT}

La reproducción de escenarios propios del mundo laboral real es esencial tanto para motivar a los y las estudiantes como para otorgarles ventajas competitivas a la hora de acceder al mercado de trabajo (Kiraly, 2005; Gutiérrez-Artacho, et al., 2016). Por otra parte, la localización de un sitio web en varios idiomas requiere de la adopción de un enfoque flexible y de la utilización de diversas herramientas y métodos que deberán asentarse en un robusto flujo de trabajo (Corte, 2002). En consecuencia, para simular un entorno de trabajo real que se adapte a la naturaleza dinámica de esta disciplina, la formación debe involucrar una amplia gama de actividades basadas en el trabajo en equipo y en el aprendizaje por proyectos (Robinson et al., 2016a).

El Modelo Profesional para la Didáctica de la Traducción (MPDT o PATT, Professional Approach to Translator Training) es un modelo didáctico creado por el grupo AulaInt, el cual se ha ido revisando y adaptando a lo largo del tiempo para adaptarse a las nuevas necesidades del ámbito de la traducción. En este caso, el modelo se ha revisado y adaptado para adaptarse a las nuevas demandas del perfil profesional de localizador web.

Esta propuesta se acerca al mundo empresarial real a través de una simulación de trabajo en equipo como si se tratase de un encargo real. Este modelo no solo tiene en cuenta la competencia comunicativa completa, sino que también abarca los conocimientos y habilidades relacionados con el uso de las nuevas tecnologías. Además, mediante su aplicación, se busca la coordinación entre las 
diferentes asignaturas del Grado en Traducción e Interpretación con el fin de que el alumnado obtenga una visión completa del proceso profesional manteniendo a su vez un enfoque interdisciplinar.

De este modo, nuestra propuesta se aproxima a los objetivos de la Declaración de Bolonia y del Proyecto Tuning, puesto que pretende ofrecer una enseñanza orientada a la profesión que facilite la empleabilidad a la vez que se buscan puntos comunes entre el ámbito académico y el profesional.

Nuestro modelo está centrado en los y las estudiantes y su volumen de trabajo, además de fomentar la comprensión de los objetivos de aprendizaje en todos los participantes. Por otro lado, contribuye a aproximar los modos habituales de producción a las aulas y apoya la interiorización de rutinas de trabajo reales, que después otorgarán una cierta ventaja competitiva en el acceso al mercado de trabajo.

Los objetivos del modelo didáctico que proponemos se encaminan a: a) familiarizar al estudiante de traducción con el método de trabajo del mercado profesional. Así, el o la estudiante puede realizar labores relacionadas con la documentación, la terminología, la localización/traducción, la revisión y la maquetación así como la gestión de proyectos, un perfil que no suele estar incluido en la docencia pero que es cada vez más importante en el mercado; b) potenciar el teletrabajo en equipo, el autoaprendizaje y la interdisciplinariedad; c) ofrecer un marco de trabajo y las herramientas útiles a los y las docentes e investigadores para la incorporación de las nuevas tecnologías al aula y, de igual modo, ofrecerles la posibilidad de reproducir el medio de trabajo profesional en el entorno académico; d) promover la coordinación entre asignaturas en la docencia universitaria del Grado en Traducción e Interpretación.

Al llevar este modelo a la práctica los alumnos y alumnas se organizan en equipos de trabajo de manera que pueden adoptar el rol correspondiente dentro de su equipo (documentalista, terminólogo, etc.). En cada equipo la secuencia de tareas asumidas por cada componente sigue un orden ascendente y se resume a continuación (Ver Figura 1):

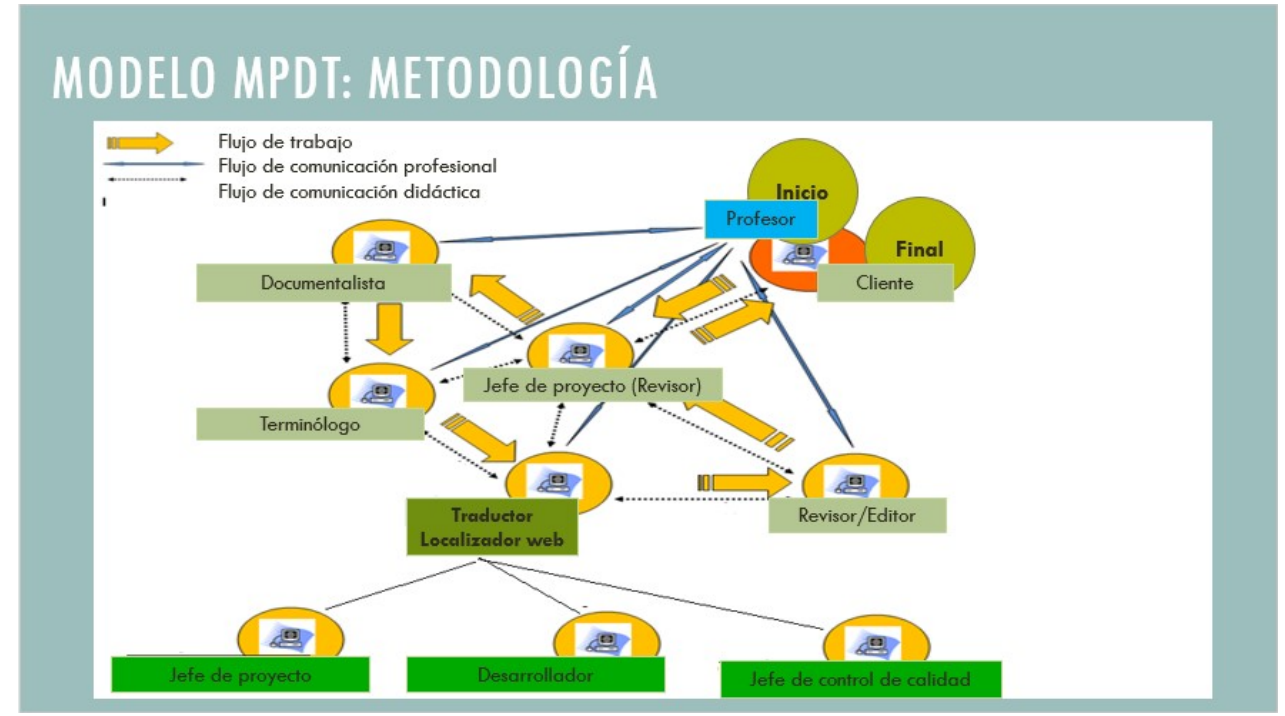

Figura 1. Modelo MPDT adaptado a la formación en la localización web

1. El o la estudiante encargado de la búsqueda documental lleva a cabo la localización de textos paralelos y de textos relacionados con el encargo.

2. El o la estudiante encargado de la búsqueda terminológica realiza una recopilación sistemática de términos pertinentes a partir de la documentación encontrada por el eslabón precedente de la cadena y de las fuentes terminológicas que considere oportuno utilizar. El sistema conceptual realizado les permite estructurar el conocimiento del texto para facilitar su adquisición. 
3. El o la estudiante encargado de la traducción/localización lleva a cabo la labor traductora y localizadora propiamente dicha, apoyándose en los resultados obtenidos en las tareas anteriores. Además, debe coordinarse con otros agentes externos a la cadena de traducción como el jefe del proyecto, los desarrolladores de softwares y los controladores de calidad.

4. Los y las estudiantes que ejercen de revisores y maquetadores realizan la revisión textual de la traducción y, posteriormente, dan el formato correspondiente al texto para su presentación como producto final acorde con las pautas dictadas en el encargo.

5. El jefe o jefa de proyecto de traducción asigna las tareas, coordina el trabajo de los miembros del equipo, establece plazos y vela por el buen desarrollo del proyecto.

De esta forma, hay dos componentes que realizan trabajos previos a la realización de la traducción, uno más que aborda la labor centrada en la traducción/localización y otros dos que efectúan las operaciones necesarias con posterioridad a la traducción/localización. El cuarto eslabón, el revisor o revisora, que ejerce de jefe o jefa de proyecto, se encarga además de asignar objetivos y plazos y de establecer el marco de actuación, como la interpretación del encargo, además de realizar un seguimiento del trabajo de los miembros del equipo para garantizar su funcionamiento.

\section{Resultados}

La necesidad de incluir formación específica sobre localización en el currículum de las enseñanzas regladas de Traducción resulta innegable tras revisar las percepciones de algunos de los autores más relevantes en este ámbito. Los autores señalan que las TIC constituyen una pieza clave en este proceso y, por tanto, la formación en localización debe siempre tener esto presente. Por tanto, a los y las estudiantes se les debe proporcionar tanto un conocimiento básico sobre informática que debe enriquecerse con conocimientos específicos tanto de las herramientas usadas durante el proceso de traducción - y que pueden emplearse en el proceso de localización- como de herramientas y aplicaciones específicas del ámbito de la localización.

Sin embargo, no se puede perder de vista que la localización va más allá de aunar la traducción y las TIC, sino que hay un componente cultural que debe ser tenido en cuenta durante todo el proceso. De este modo, los y las estudiantes de traducción deben poseer una competencia comunicativa completa que debe ser adquirida a lo largo de todo su proceso formativo.

Por otro lado, la entrada en vigor de la Declaración de Bolonia supone que el contenido del currículum de los sistemas de la educación superior debe verse adaptado para cumplir con los objetivos establecidos, encaminados principalmente a fomentar la empleabilidad de los y las estudiantes.

En consecuencia, este artículo presenta el modelo didáctico MDPT adaptado a la formación de los profesionales de la localización con el fin de dar respuesta, por un lado, a las nuevas habilidades requeridas por este nuevo perfil profesional -el dominio de las TIC y la competencia comunicativa completa- y, por otro lado, a los objetivos establecidos en la Declaración de Bolonia, entre los que se incluye la orientación hacia la incorporación al ámbito profesional y la interdisciplinariedad y la transversalidad con otras áreas de conocimiento.

\section{Discusión}

En primer lugar, el modelo MPDT adaptado a la formación en localización web se aproxima a los objetivos de la Declaración de Bolonia puesto que ofrece una enseñanza orientada a la profesión. Al tener una experiencia cercana a una situación profesional real los y las estudiantes tienen ventajas competitivas a la hora de acceder al mercado laboral de la traducción y la localización puesto que interiorizan rutinas de trabajo reales. 
Además, los alumnos y alumnas adquieren competencias como el trabajo en equipo, ya que se organizan en grupos de trabajo; el autoaprendizaje, puesto que cada alumno o alumna tiene que responsabilizarse de una parte del proceso de localización y, aunque los y las estudiantes cuentan con la asistencia de los y las docentes, ellos mismos deben responder a las demandas del eslabón siguiente adaptando sus concepciones; la interdisciplinariedad, puesto que los alumnos y alumnas adoptan roles de diferentes áreas relacionadas con la traducción como la documentación, la maquetación o la terminología. Así, el alumnado se puede hacer una idea de cada una de las tareas de los diferentes eslabones de la cadena de producción en relación con el conjunto durante su estancia en la Universidad. Por último, al simular una situación de trabajo real se fomenta el uso de las nuevas tecnologías en el aula al emplearse softwares específicos y herramientas que faciliten las relaciones profesionales por medios electrónicos.

\section{Referencias bibliográficas}

Anastasiou, D. (2010). Survey on the Use of XLIFF in Localisation Industry and Academia. En G. Budin, L. Romary, T. Declerck, \& P. Wittenburg (eds.). Proceedings of Language Resource and Language Technology Standards-State of the Art, Emerging Needs, and Future Developments Workshop, 7th International Conference on Language Resources and Evaluation (LREC) (pp. 50-53). Malta: ELDA.

Arevalillo-Doval, J.J. (2000). La Localización: Concepto, Nuevas Tecnologías Y Requisitos Del Nuevo Traductor De Informática. En IV Encuentros Alcalainos de Traducción (pp. 1-9). Alcalá de Henares.

Austermühl, F. (2006). Training translators to localize, En A. Pym, A. Perekrestkenko, \& B. Starink (Eds.), Translation Technology and Its Teaching (pp. 69-81). Tarragona: Inter Cultural Studies Group.

Cadieux, P, \& Esselink, B. (2014). GILT: Globalization, Internationalization, Localization, Translation. Globalization Insider, 11(5), 1-5.

Consejo de Europa (2001). Marco Común Europeo de Referencia para las Lenguas: aprendizaje, enseñanza, evaluación. Madrid: Instituto Cervantes - Ministerio de Educación Cultura y Deporte - Anaya. http://cvc.cervantes.es/obref/marco.

Corte, N. (2002). Localización e internacionalización de sitios web. Revista Tradumática, 1, 1-8.

Drouin, P. (2006). Training for localization (replies to a questionnaire). En A. Pym, A. Perekrestenko, \& B. Starink (Eds). Translation technology and its teaching (pp. 39-44). Tarragona: Inter Cultural Studies Group.

Dunne, K. (2006). Perspectives on Localization. Amsterdam: John Benjamins Publishing.

García-Saavedra, M.T. (2002). ¿Traduces o localizas? La localización, futuro y presente de la traducción. En L. González \& Pollux Hernúñez (Coords.), El español, lengua de traducción. I Congreso Internacional (pp. 409-23). Ciudad Real: EsLetra.

Gaspari, F., H. Almaghout, \& S. Doherty (2016). A survey of machine translation competences: Insights for translation technology educators and practitioners. Perspectives: Studies in Translatology, 23(3), 333-58. doi: 10.1080/0907676X.2014.979842.

Gutiérrez-Artacho, J. \& Olvera-Lobo, M.D. (2013). Los procesos de enseñanza-aprendizaje de la traducción especializada desde la perspectiva profesional. En Jornadas de Traducción bispanorusas. Moscú, Rusia.

Gutiérrez-Artacho J., Olvera-Lobo, M.D., \& Hunt-Gómez, C.I. (2016). Incorporación de las nuevas tecnologías de la innovación y un modelo didáctico adaptado a la formación en Traducción e Interpretación. En E. López-Meneses, D Cobos Sanchiz, A.H. Martín Padilla, L. Molina García y A. Jaén Martínez (Dirs.), Actas del III Congreso Virtual Internacional sobre Innovación Pedagógica y Praxis Educativa. Innovagogia 2016. Sevilla: Colectivo Docente INNOVAGOGÍA y AFOE Formación. 
Gutiérrez-Artacho, J., \& Olvera-Lobo, M.D. (2016). Teaching methodologies for new professional profiles in the translation market: web locators. En Gómez Chova, L, López Martínez, A., \& Candel Torres, I. (Eds.), ICERI2016 Proceedings: 9th annual International Conference of Education, Research and Innovation, 14-16 de noviembre (pp. 3384-3392). Sevilla-Valencia: IATED Academy. DOI: 10.21125/iceri.2016.1791.

Gutiérrez-Artacho, J., \& Olvera-Lobo, M.D. (2017). El método Delphi como herramienta de evaluación en la formación en nuevos perfiles profesionales de la traducción. En $5^{\circ}$ Congreso Internacional Entreculturas de Traducción e Interpretación, 27-30 de junio. Málaga: Universidad de Málaga.

Gutiérrez-Artacho, J., Olvera-Lobo, M.D. (2017). El uso del método Delphi como herramienta de evaluación consensuada en la didáctica de la traducción: el perfil del traductor-localizador. En Del Valle, M.E. (Coord.), Congreso CUICIID 2017: Congreso Universitario Internacional sobre la comunicación en la profesión y en la Universidad de hoy: Contenidos, Investigación, Innovación y Docencia, 25-26 de octubre (pp. 226-240). Disponible en: https://bit.ly/2xafRTJ.

Gutiérrez-Artacho, J., \& Olvera-Lobo, M.D. (2018). Computer tools in the teaching of translators as website localization professionals. En INTED2018: 12th International Technology, Education and Development Conference. Proceedings, 5-7 de marzo (pp. 1169-1175). Valencia: INTED Academy.

Hussein, A.S. (2007). The Impact of Translation Market on Translator Training. Al-Mustansiriya Journal of Arts, 47, 1-23.

Jiménez-Crespo, M.A, \& Tercedor, M. (2011). Applying corpus data to define needs in web localization training. Meta Journal des Traducteurs, 56(4), 998-1021. doi: 10.7202/1011264ar

Kiraly, D. (2005). Learning: A Case for Situated Translation Project-Based Learning: A Case for Situated Translation. Meta, Journal des Traducteurs, 50, 1098-1111. doi: 10.7202/012063.

LISA. 2003. The Localization Industry Primer. Disponible en: https://bit.ly/2XwkZAi.

Oasis XLIFF (2003). Xliff 1.1. A white paper on version 1.1 of the XML Localisation Interchange File Format (XLIFF). Disponible en: https://bit.ly/2ZIqreO.

O'Hagan, M. (2006). Training for localization (replies to a questionnaire). En En A. Pym, A. Perekrestenko, \& B. Starink (Eds). Translation technology and its teaching (pp. 39-44). Tarragona: Inter Cultural Studies Group.

Olvera Lobo, M.D., Castro Prieto, M.R., Robinson, B., Quero, E., Muñoz Martín, R., Muñoz Raya, E., Murillo, M., Senso, J.A., Vargas-Quesada, B., \& Domínguez-López, C. (2005). Translator training and modern market demands. Perspectives, 13(2)., 132-42. doi:10.1080/09076760508668982.

Olvera-Lobo, M.D., Castro-Prieto, R.M., Quero-Gervilla, E., Muñoz-Martín, R., Muñoz Raya, E., Murillo-Melero, M., Robinson, B., \& Senso-Ruiz, J. (2008). Collaborative Work Learning in Higher Education. En M.M. Putnik \& G. D; Cunha (Eds.), Encyclopedia of Networked and Virtual Organizations (pp. 261-268). Hershey: Idea Group.

Olvera-Lobo, M.D., \& Gutiérrez-Artacho, J. (2011). Initiatives and evaluation of teaching-learning process in translation training. En M. Garant (Ed.), Current trends in translation teaching and learning (pp. 79-95). Helsinky: University.

Olvera-Lobo, M.D., \& Gutiérrez-Artacho, J. (2017a). Los procesos de enseñanza-aprendizaje de la traducción desde una perspectiva profesional e interdisciplinar: la localización web como nuevo mercado. En XV Jornadas Hispano-Rusas de Traducción e Interpretación. Universidad Estatal Lingüistica de Moscú, Moscú, Rusia.

Olvera-Lobo, M.D., \& Gutiérrez-Artacho, J. (2017b.) Training versus profession: from traslation to web location. En Proceedings of INTED2017 Conference (pp. 5461-5469). Valencia: IATED.

Olvera-Lobo, M.D., Quero-Gervilla, E., Robinson, B, Senso-Ruiz, J.A., Castro-Prieto, M.R., MuñozMartín, R., Muñoz-Raya, E., \& Murillo-Melero, M. (2007a). A professional approach to translator training (PATT). Meta, Journal des Traducteurs, 52(4), 517-28. doi: 10.7202/016736ar.

Olvera-Lobo, M.D., Robinson, B.J., Senso, J.A., Muñoz-Martín, R., Muñoz-Raya, E., Murillo-Melero, M., Quero-Gervilla, E., Castro-Prieto, M.R., \& Conde-Ruano, T. (2007b). Student 
satisfaction and perceived improvement with a Web-based collaborative work platform. Perspectives: Studies in Translatology, 15(2), 106-22. doi: 10.1080/13670050802153848.

Olvera-Lobo, M.D., Robinson, B., Senso, J.A., Muñoz-Martín, R., Muñoz-Raya, E., Murillo Melero, M., Quero-Gervilla, E., Castro-Prieto, M.R., \& Conde-Ruano, T. (2009a). Teleworking and collaborative work environments in translation training. Babel: International Journal of Translation, 55(2), 165-80. doi: 10.1075/babel.55.2.05olv.

Olvera-Lobo, M.D., Quero-Gervilla, E., Robinson, B., Senso-Ruiz, J.A., Castro-Prieto, M.R., MuñozMartín, R., Muñoz-Raya, E., \& Murillo-Melero, M. (2009b). Predstavlenie modeli distantsionnoj raboty dlja vnedrenia $\mathrm{v}$ praktiku prepodavania perevoda $\mathrm{v}$ sootvetsvii $\mathrm{s}$ trebovaniami Bolonskoj deklaratsii. Vestnik MGU, Seria no 22. Teoria Perevoda, 1, 62-75.

Olvera-Lobo, M.D., Castro-Prieto, R.M., Muñoz Martín, R., Robinson, B., \& Villena, I. (2003). Nuevas tecnologías y didáctica de la traducción. Innovación docente vía Internet. Revista Universitaria ICE, 21, 97-108.

Olvera-Lobo, M.D., Castro-Prieto, R.M., Muñoz Martín, R., Robinson, B., \& Villena, I. (2003). Aula virtual de traducción: AULA.INT. Proyecto de innovación docente. En I Congreso Internacional de la Asociación Ibérica de Estudios de Traducción e Interpretación (AIETI) (pp. 471-489). Granada, España.

Olvera-Lobo, M.D., Gutiérrez-Artacho, J., \& Robinson, B.J. (2013). Acciones formativas Web 2.0: el grado en traducción e interpretación como banco de pruebas. En VI Congreso Internacional de la Asociación Ibérica de Estudios de Traducción e Interpretación. Las Palmas de Gran Canaria, España.

Olvera-Lobo, M.D., Quero-Gervilla, E., Robinson, B., Senso-Ruiz, J.A., Castro-Prieto, M.R., MuñozMartín, R., Muñoz-Raya, E., \& Murillo-Melero, M. (2010). Presentation of a distance training model for introduction into the practice of teaching translation according to the requirements of the Bologna declaration. Vestnik MGU. Seria no 26. Pedagogical Sciences, 605, 196-208.

Olvera-Lobo, M.D., \& Gutiérrez-Artacho, J. (2014a). Academic use of custom social networks in translation training. Perspectives: Studies in Translatology, 22(2), 282-89. doi: 10.1080/0907676X.2012.706310.

Olvera-Lobo, M.D., \& Gutiérrez-Artacho, J. (2014b). Herramientas de software libre en la docencia de la traducción (PID 12-139). En Innovacion docente y buenas prácticas en la Universidad de Granada (pp. 710-722). Granada: Editorial Universidad de Granada.

Olvera-Lobo, M.D., \& Gutiérrez-Artacho, J. (2014c). Web 2.0 Technologies in the translator training: Aulalint. En Aulalint. 6th International Conference on Education and New Learning Technologies (pp. 1562-1569). Barcelona, España.

Perez-Cabrera, M.J. (2012). Aspectos técnicos y metodológicos de la localización de contenido web 1. Odisea, 13, 189-207. doi: 10.25115/odisea.v0i13.246.

Pym, A. (2013) Localization, Training, and Instrumentalization. Tarragona: Universitat Rovira i Virgili.

Raya, R. (2004a). Maxprograms - XML in Localisation: A practical analysis. Disponible en: https://bit.ly/2Xy3VtH.

Raya, R. (2004b). Maxprograms - Use XLIFF to Translate Documents. Disponible en: https://bit.ly/2ZLQR1n.

Robinson, B.J., Olvera-Lobo, M.D., \& Gutiérrez-Artacho, J. (2015). Trainee translators' perceptions of cooperative teamwork. En G. Corpas Pastor (Ed.), AIETI7 (Asociación Ibérica de Estudios de Traducción e Interpretación): Nuevos horizontes en los estudios de traducción e interpretación, (pp. 37593). Ginebra: Tradulex.

Robinson, B. J., Olvera-Lobo, M. D., \& Gutiérrez-Artacho, J. (2016a). After Bologna: Learner- and Competence-Centred Translator Training for "Digital Natives". En Martín de León, C., \& González-Ruiz, V. (Eds.), From the Lab to the Classroom and Back. Again: Perspectives on Translation and Interpreting Training. New Trends in Translation Studies Series. Frankfurt am Main: Peter Lang.

Robinson, B.; Olvera-Lobo, M.D., \& Gutiérrez-Artacho, J. (2016b). The Professional Approach to Translator Training Revisited. En From the Lab to the Classroom and Back. Again: Perspectives on 
84 Juncal Gutiérrez-Artacho, María-Dolores Olvera-Lobo \& Irene Rivera-Trigueros

Translation and Interpreting Training. New Trends in Translation Studies Series. Frankfurt am Main: Peter Lang

Sandrini, P. (2015). Website Localization and Translation. En MuTra 2005 - Challenges of Multidimensional Translation: Conference Proceedings. Saarbrücken, Austria.

Para citar este artículo

Gutiérrez-Artacho, J., Olvera-Lobo, M.D., \& Rivera-Trigueros, I. (2019). Competencia comunicativa y nuevas tecnologías en el proceso de localización web: modelo MDPT para la formación de profesionales en localización. Revista Fuentes, 21(1), 73-84. [Fecha de consulta: dd/mm/aa]. doi: 10.12795/revistafuentes.2019.v21.i1.05 